\title{
Pre-outburst observations of Nova Del 2013 from Pan-STARRS 1 (Research Note)
}

\author{
N. R. Deacon ${ }^{1}$, D. W. Hoard ${ }^{1, \star}$, E. A. Magnier ${ }^{2}$, Y. S. Jadhav ${ }^{1,3}$, M. Huber ${ }^{2}$, K. C. Chambers ${ }^{2}$, H. Flewelling ${ }^{2}$, \\ K. W. Hodapp ${ }^{4}$, N. Kaiser ${ }^{2}$, R. P. Kudritzki ${ }^{2}$, N. Metcalfe ${ }^{5}$, and C. Waters ${ }^{2}$ \\ 1 Max Planck Institute for Astronomy, Königstuhl 17, 69117 Heidelberg, Germany \\ e-mail: deacon@mpia.de \\ 2 Institute for Astronomy, University of Hawai'i, 2680 Woodlawn Drive, HI 96822, USA \\ 3 Dept. of Physics \& Astronomy, College of Arts \& Sciences, Clippinger Labs 251B, Ohio University, Athens OH 45701, USA \\ ${ }^{4}$ Department of Physics, University of Durham, South Road, Durham DH1 3LE, UK \\ 5 Institute for Astronomy, University of Hawai'i, 640 N. Aohoku Place, Hilo HI 96720, USA
}

Received 18 February 2014 / Accepted 24 February 2014

ABSTRACT

\begin{abstract}
Context. Nova Delphini 2013 was identified on the 14th of August 2013 and eventually rose to be a naked eye object.
Aims. We sought to study the behaviour of the object in the run-up to outburst and to compare it to the pre-outburst photometric characteristics of other novae.

Methods. We searched the Pan-STARRS 1 datastore to identify pre-outburst photometry of Nova Del 2013 and identified twenty-four observations in the 1.2 years before outburst.

Results. The progenitor of Nova Delphini showed variability of a few tenths of a magnitude but did not brighten significantly in comparison with archival plate photometry. We also found that the object did not vary significantly on the approximately half hour timescale between pairs of Pan-STARRS 1 observations.
\end{abstract}

Key words. novae, cataclysmic variables - surveys

\section{Introduction}

Nova Delphini 2013 (=V339 Del) was first identified as a bright 6.8 mag source by Koichi Itagaki on the 14th of August 2013 and announced in AAVSO Alert Notice 489 (Waagen 2013). Since then this object has been extensively monitored using radio continuum (Roy et al. 2013), near-infrared (Stringfellow et al. 2013), optical (Darnley \& Bode 2013; Tarasova 2013), X-ray (with Page et al. 2013 first detecting emission 36 days after discovery) and gamma-ray (Hays et al. 2013) observations. Initially the nova exhibited strong P Cygni profiles in the Balmer and He I lines (Tarasova 2013), these profiles disappeared from the hydrogen lines on the 19th of August (Darnley \& Bode 2013) but remained for other species (He I, O I, Fe II).

The progenitor of this nova has been identified as USNOB1.0 1107-0509795, which had an unfiltered magnitude of 17.1 on the 14th of May 2013 (Denisenko \& Masi 2013). Wren et al. (2013) report a pre-discovery detection as the nova was brightening. Data on this object prior to outburst remain relatively scarce. Denisenko reports a counterpart in the UV from GALEX while there are a handful of detections in POSS photographic plate catalogues. Tang et al. (2013) report three pairs of $\mathrm{H} \alpha$ on/off measurements but find no strong evidence that the progenitor is an $\mathrm{H} \alpha$ emitter. Munari \& Henden (2013) identified the progenitor in Asiago photographic plate data, finding 12 detections and 13 upper limits on the object's brightness. Over the course of three years of observations from 1979 to 1982, the progenitor showed variation in the $B$-band with a total amplitude of 0.9 mag. Munari \& Henden (2013) also stacked three

\footnotetext{
* Visiting Scientist, MPIA.
}

observations taken by the APASS survey ${ }^{1}$ in April 2012 to provide additional $B$ and $V$ photometry.

\subsection{Pre-outburst observations of novae}

Robinson (1975) presented a landmark study of pre- and postoutburst data for novae from archival photographic plates. Out of 18 targets with both pre- and post-outburst magnitude measurements, he identified only one object (BT Monocerotis) with significantly different brightnesses. Out of 12 targets with wellsampled pre-outburst light curves, five showed an apparent increase in luminosity in the few years before outburst, and two showed significantly increased variability in the lead-up to their outbursts.

These claims were later re-examined by Collazzi et al. (2009), who combined subsequent remeasurements of plate data both from their own work and from other sources (whereas Robinson 1975 relied on the available pool of published photometry from the plates, which can suffer from systematic calibration errors up to $\gtrsim 1 \mathrm{mag}$ ). They concluded that BT Mon actually showed no significant change between its pre- and post-outburst brightness and did not reproduce four of the five claims of preoutburst brightening. They confirmed that the one remaining object from the Robinson (1975) pre-outburst brightening sample (V533 Herculis) did, in fact, increase in brightness by $\sim 1.3 \mathrm{mag}$ in the $\sim 1.5$ years leading up to outburst. Collazzi et al. (2009) identified an additional nova (V1500 Cygni) that showed preoutburst brightening, rising nearly $7 \mathrm{mag}$ in the month before its

\footnotetext{
1 http://www. aavso.org/apass
} 
outburst $^{2}$. In addition, Collazzi et al. (2009) found no case for a class of novae with a significant increase in variability leading up to the outburst, noting that one such object claimed by Robinson (1975) (RR Telescopii) has since been found to be a symbiotic star and that the other (V446 Herculis) actually did not exhibit any change in its variability behaviour before and after outburst. They also note that changes in variability amplitude in general are characteristics of novae in quiescence and may not be associated with the outburst - they conclude that none of the novae they examined showed a significant change in variability that could be linked to the outburst. It should be noted that Collazzi et al. (2009) also found a pre-outburst dip in the recurrent nova $\mathrm{T}$ CrB. Recent additional archival photometry unearthed by Schaefer (2014) indicates that this object was $\sim 1$ mag brighter in the 8 years leading up to this brief pre-erruption dip. Schaefer et al. (2013) also report a brief, temporary brightening of $\sim 1$ mag of another recurrent nova, T Pyx, prior to outburst.

The total brightness of cataclysmic variables (CVs) with accretion disks - of which classical novae are a subset - are dominated by accretion-generated luminosity over a wide range of the electromagnetic spectrum (from the near-IR to the far-UV). As such, variability in the long-term ${ }^{3}$ mean brightness is directly proportional to changes in the mass transfer rate from the low mass donor star, through the disk, and onto the white dwarf (WD) primary star. The origin of a classical nova outburst is a thermonuclear runaway in the thin layer of accreted hydrogen-rich matter on the surface of the WD. The total mass in this layer required to trigger the runaway is a few $\times 10^{-3} M_{\odot}$ for a WD with $M_{\mathrm{WD}}=0.6 M_{\odot}$ (i.e., the average WD mass) to a few $\times 10^{-5} M_{\odot}$ for WDs approaching the Chandrasekhar mass (see Warner 2003, and references therein). Even under the optimistic assumption of a persistent high CV mass transfer rate of $\sim 10^{-9} M_{\odot} \mathrm{yr}^{-1}$, the timescale to grow this accreted envelope to the critical mass is $\gtrsim 10^{4}-10^{6} \mathrm{yr}$.

This is the root of the reason that Collazzi et al. (2009) found Robinson's conclusion that almost $50 \%$ of novae show a preoutburst brightness increase to be "unsettling". They discuss in some detail the arguments against a corresponding pre-outburst increase in the mass transfer rate being the "last straw" that triggers the nova outburst. In summary, (a) the amount of extra mass accumulated during the rise in brightness is miniscule compared to the total mass required to trigger the outburst; and (b) the energy flux carried by the extra accreted matter produces an insignificant increase in the temperature at the base of the accumulated layer on the WD. The result of Collazzi et al. (2009), two out of 22 novae $(<10 \%)$ displaying a pre-outburst rise in brightness, might well be less unsettling, but it is still problematic: as they note, the close proximity to the nova outbursts in both V533 Her and V1500 Cyg suggests that the pre-outburst brightness increases are somehow causally connected to the outbursts, although they cannot have directly triggered the outbursts.

\footnotetext{
2 Although post-outburst behaviour is not explicitly the topic of this paper, we note that V1500 Cygni was also one of the five (out of 30) novae that Collazzi et al. (2009) found to have brightened by more than a factor of 10 after outburst, while the remaining majority of novae had essentially no change in quiescent brightness across the outburst.

3 "Long-term", in this case, is relative since it might easily be measured in units of the CV's orbital period. At typical values of $\sim 1-10 \mathrm{~h}$, even many orbital cycles span an interval that is, in an absolute sense, not very long. CVs, of course, famously display a wide range of short timescale, low amplitude variability - "flickering" - but this is likely related to the effects of viscous turbulence in the disk and/or accretion stream and not to actual changes in the mean mass transfer rate from the donor star (Scaringi 2014).
}

In the majority of classical novae, it appears, the outburst should arrive as a surprise, without previous announcement in the light curve. It is the currently unexplained exceptions to this situation, like V533 Her and V1500 Cyg, that might offer clues that will help to further illuminate the nova outburst mechanism. Similarly, although both Robinson (1975) and Collazzi et al. (2009) agree that the majority of novae have the same preand post-outburst mean quiescent brightness (implying that the outburst does not significantly alter the mass transfer process in the CV), the latter found that $\sim 15 \%$ of novae do show a postoutburst increase in brightness of $>2.5$ mag. Again, the few exceptions to the majority behaviour might offer the best insight into the nova outburst mechanism. Obtaining post-outburst data on novae is easy, but for pre-outburst data, we must typically rely on serendipitous observations. In this paper we describe preoutburst photometry from Pan-STARRS 1 for Nova Del 2013.

\section{Observations}

\subsection{Pan-STARRS 1 observations}

Pan-STARRS 1 is a $1.8 \mathrm{~m}$ high-etendue survey telescope situated on Haleakala on Maui in the Hawaiian Islands (Kaiser et al. 2002). Approximately $50 \%$ of the time on this telescope is devoted to the $3 \pi$ survey, which is covering the sky north of $-30^{\circ}$ in five filters $\left(g_{P 1}, r_{P 1}, i_{P 1}, z_{P 1}\right.$ and $y_{P 1}$ with effective wavelengths of 481, 617, 752, 866 and $962 \mathrm{~nm}$ respectively; Tonry et al. 2012) with six pairs of observations per filter in each area of sky over the course of the survey. The survey photometric reference system and calibration are described by Magnier et al. (2013) and Schlafly et al. (2012) respectively. The $3 \pi$ survey strategy is designed to accommodate various science and observing constraints. Observations in $z_{P 1}$ and $y_{P 1}$ are taken 3-4 months before opposition to maximise the parallax factor for late-type objects. The two pairs of observations in each of $g_{P 1}, r_{P 1}$ and $i_{P 1}$ are usually taken in one lunation close to opposition. Observations in the same filter are taken in pairs separated by approximately $25 \mathrm{~min}$. These pairs are used for asteroid detection but are also useful in the study of CVs, spanning a significant portion of the typical short period variability timescales for such objects. Hence variability between the observations in a pair is a characteristic of CVs (Jadhav et al., in prep., 2014).

We extracted all data for Nova Delphini 2013 from the Pan-STARRS 1 data store. In order to plot only points with reliable photometry we followed the suggestion of Morganson et al. (2012) and only selected observations with a point spread function (PSF) quality factor (the fraction of pixels from the object PSF, which were not saturated and did not fall on bad pixels or chip-gaps) greater than 0.8 . These are listed in Table 1 .

\subsection{Other photometry}

We extracted observations from other sources by searching various astronomical archives (see Table 2). Our main source comes from the SuperCOSMOS Sky Survey (Hambly et al. 2001b) scans on Palomar POSS plates. While there are no errors quoted for the SuperCOSMOS fields, Hambly et al. (2001a) quote approximate errors for UKST filters as a function of magnitude. Hence for the POSS-II $R_{F}$ point the approximate error will be $0.14 \mathrm{mag}, 0.34 \mathrm{mag}$ in POSS-II $B_{J}$ and $0.17 \mathrm{mag}$. in POSS-II $I_{N}$. These datapoints are in a system on the Vega scale; for our purposes we converted these using the Vega-AB corrections of Blanton \& Roweis (2007). Note that these transformations are not for exactly the same filter set as the 


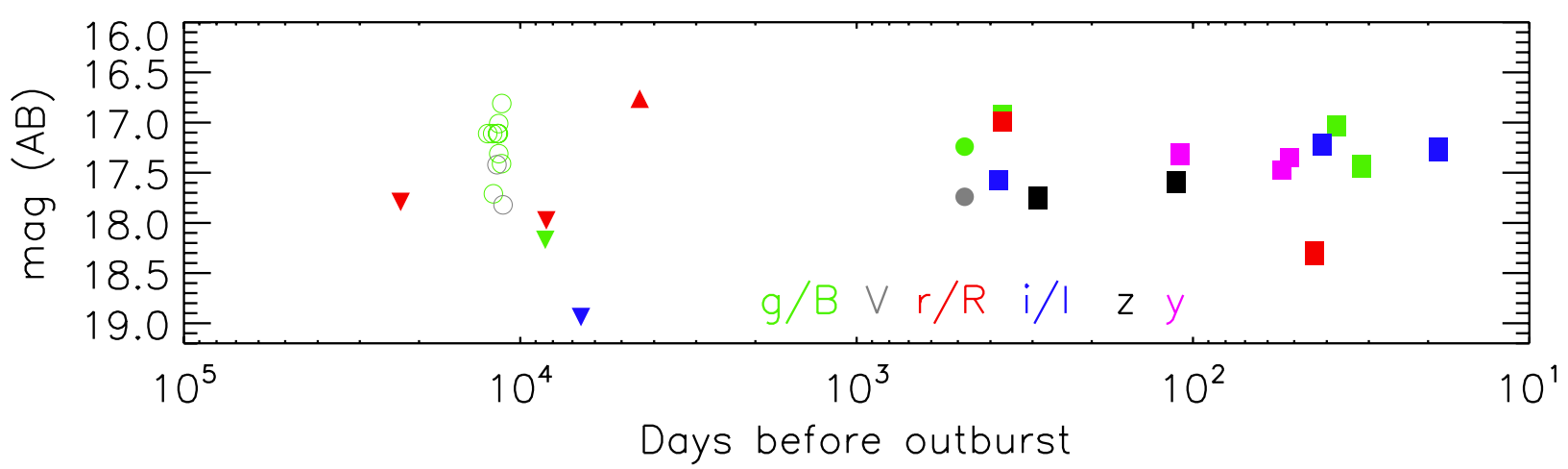

Fig. 1. Pre-outburst photometry for Nova Del 2013. Square points are from Pan-STARRS 1, downward facing triangles from plate data, upward facing triangles from the Carlsberg Meridian catalogue, filled circles are APASS data and points marked with open circles come from Asiago photographic plates.

Table 1. Photometry of Nova Del 2013 from Pan-STARRS 1.

\begin{tabular}{lccc}
\hline \hline Magnitude $(A B)$ & psf_qf & Filter & JD \\
\hline $17.569 \pm 0.029$ & 0.998 & $i_{P 1}$ & 2456138.973 \\
$17.583 \pm 0.033$ & 0.999 & $i_{P 1}$ & 2456138.984 \\
$16.914 \pm 0.029$ & 0.998 & $g_{P 1}$ & 2456149.811 \\
$16.940 \pm 0.024$ & 0.998 & $g_{P 1}$ & 2456149.821 \\
$16.986 \pm 0.041$ & 0.999 & $r_{P 1}$ & 2456150.783 \\
$16.999 \pm 0.047$ & 0.998 & $r_{P 1}$ & 2456150.794 \\
$17.733 \pm 0.027$ & 0.998 & $z_{P 1}$ & 2456228.763 \\
$17.770 \pm 0.029$ & 0.999 & $z_{P 1}$ & 2456228.774 \\
$17.576 \pm 0.027$ & 0.999 & $z_{P 1}$ & 2456406.089 \\
$17.603 \pm 0.022$ & 0.999 & $z_{P 1}$ & 2456406.101 \\
$17.297 \pm 0.024$ & 0.998 & $y_{P 1}$ & 2456409.103 \\
$17.331 \pm 0.022$ & 0.999 & $y_{P 1}$ & 2456409.115 \\
$17.474 \pm 0.029$ & 0.999 & $y_{P 1}$ & 2456464.123 \\
$17.349 \pm 0.022$ & 0.997 & $y_{P 1}$ & 2456466.984 \\
$18.325 \pm 0.022$ & 0.998 & $r_{P 1}$ & 2456475.070 \\
$18.278 \pm 0.020$ & 0.998 & $r_{P 1}$ & 2456475.080 \\
$17.206 \pm 0.031$ & 0.998 & $i_{P 1}$ & 2456477.076 \\
$17.236 \pm 0.046$ & 0.997 & $i_{P 1}$ & 2456477.087 \\
$17.040 \pm 0.042$ & 0.999 & $g_{P 1}$ & 2456481.046 \\
$17.020 \pm 0.026$ & 0.997 & $g_{P 1}$ & 2456481.056 \\
$17.414 \pm 0.030$ & 0.999 & $g_{P 1}$ & 2456486.869 \\
$17.451 \pm 0.034$ & 0.998 & $g_{P 1}$ & 2456486.879 \\
$17.244 \pm 0.031$ & 0.998 & $i_{P 1}$ & 2456499.872 \\
$17.287 \pm 0.026$ & 0.998 & $i_{P 1}$ & 2456499.879 \\
$12.831 \pm 0.020$ & 0.886 & $y_{P 1}$ & 2456565.704 \\
\hline & & &
\end{tabular}

Notes. The psf_qf parameter describes the fraction of pixels from the object PSF, which were not saturated and did not fall on bad pixels or chip-gaps. ${ }^{(\dagger)}$ This post-outburst datapoint has a slightly low psf_qf and is close to the saturation limit in the $y_{P 1}$ band. Hence it should be considered potentially unreliable.

POSS plates but are the closest available. We applied the same process to the APASS and Asiago photometry from Munari \& Henden (2013). The Asiago data do not have formal errors for each observation but Munari \& Henden (2013) quote an error of $0.1 \mathrm{mag}$ for all datapoints. All of the available broadband preoutburst data for Nova Del 2013 are shown in Fig. 1.

\section{Discussion}

Figure 1 shows that the progenitor of Nova Del 2013 was variable in the lead-up to its outburst. Table 3 quantifies the variability in each Pan-STARRS 1 band. There is an apparent
Table 2. Photometry of Nova Del 2013 from other sources.

\begin{tabular}{lcc}
\hline \hline Magnitude & Filter & JD \\
\hline 17.58 & POSS-I $E$ & 2433835.923 \\
17.2 & Asiago $B$ & 2444016.506 \\
17.2 & Asiago $B$ & 2444436.502 \\
17.8 & Asiago $B$ & 2444526.399 \\
17.4 & Asiago $V$ & 2444812.455 \\
17.2 & Asiago $B$ & 2444840.491 \\
17.2 & Asiago $B$ & 2444854.431 \\
17.2 & Asiago $B$ & 2444901.382 \\
17.1 & Asiago $B$ & 2444925.258 \\
17.4 & Asiago $B$ & 2444931.316 \\
17.5 & Asiago $B$ & 2445148.554 \\
16.9 & Asiago $B$ & 2445166.542 \\
17.8 & Asiago $V$ & 2445263.365 \\
18.26 & POSS-II $B_{J}$ & 2448091.863 \\
17.77 & POSS-II $R_{F}$ & 2448150.706 \\
18.49 & POSS-II $I_{N}$ & 2449917.867 \\
16.76 & CMC $r^{\prime}$ & 2452102 \\
$17.33 \pm 0.09^{\ddagger}$ & APASS $B$ & 2456040.833 \\
$17.06 \pm 0.10^{\ddagger}$ & APASS $V$ & 2456040.833 \\
\hline
\end{tabular}

Notes. The POSS plate photometry is on the Vega scale and comes from the SuperCOSMOS Sky Survey (Hambly et al. 2001b). The Carlsberg Meridian (CMC 2006) point is on the $\mathrm{AB}$ scale and the Asiago and APASS measurements are on the Vega scale. ${ }^{(\dagger)}$ The Carlsberg Meridian catalogue lists the two datapoints for the object as being nonphotometric. Additionally the epoch and magnitude are means of the

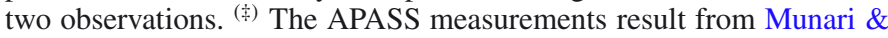
Henden (2013) stacking three observations from the nights of the 21st, 24th and 25th of April 2012. Here we assign APASS observations the mean epoch of these three dates.

Table 3. Variability of Nova Del 2013 in Pan-STARRS 1 data taken in the 1.2 years running up to the outburst.

\begin{tabular}{lccc}
\hline \hline Filter & Mean & $\sigma$ & $n_{\text {meas }}$ \\
\hline$g_{P 1}$ & 17.130 & 0.219 & 6 \\
$r_{P 1}$ & 17.647 & 0.655 & 4 \\
$i_{P 1}$ & 17.354 & 0.159 & 6 \\
$z_{P 1}$ & 16.671 & 0.082 & 4 \\
$y_{P 1}$ & 17.363 & 0.067 & 4 \\
\hline
\end{tabular}

trend of increasing variability with decreasing wavelength as expected from flickering in CVs (Bruch 1992); however, it is impossible to state with certainty if this trend is real or the 
result of sparse sampling. We also note that none of the preoutburst pairs of observations show differences between their components of more than $2 \sigma$. This indicates that the progenitor was not significantly variable on timescales of approximately half an hour. Additionally, we saw no dwarf-nova-like outbursts of a magnitude or more. While the Pan-STARRS 1 data are marginally brighter than the POSS photographic data (excluding the $I_{N}$ observation, which is close to the sensitivity limit for the $I_{N}$ plates) there is no evidence for a significant increase in brightness from less than a month to several years before the outburst, as was observed for V1500 Cyg and V533 Her, respectively (Collazzi et al. 2009). Ignoring the influence of different bandpasses and considering the data in Fig. 1 en masse, the recent Pan-STARRS 1 photometry of Nova Del 2013 is possibly $\sim 0.5$ mag brighter than the much earlier SuperCOSMOS archival observations (but not the APASS, Carlsberg or Asiago data). Even if this is a real feature and not, for example, an artifact resulting from the comparison of historic photographic photometry with modern CCD photometry, then it is within the limit of the expected level of "normal" variability based on observations of many nova light curves away from outburst (Collazzi et al. 2009). Once Nova Del 2013 fades back to quiescence, it will be illuminating to compare its post-outburst behaviour to the pre-outburst data measured in this paper.

\section{Conclusions}

We find that prior to its eruption, Nova Del 2013 did not show a significant change in photometric behaviour. Similar to most of the novae studied by Collazzi et al. (2009), the progenitor did not show a significant increase in brightness in the few years prior to outburst. The lack of significant variability between the components of Pan-STARRS 1 observation pairs indicates that the progenitor was not significantly variable on the timescale of approximately half an hour.

During the next several years, a similar amount of preoutburst photometry will be available for the majority of northern novae from the ongoing Pan-STARRS project. A number of similar ongoing and planned wide-field, temporal surveys will provide additional multi-epoch photometric coverage of nova progenitors over, collectively, a large fraction of the entire sky in both hemispheres (e.g., the Catalina Real-time Transient Survey, Drake et al. 2012; the Palomar Transient Factory, Rau et al. 2009; SkyMapper, Keller et al. 2013). Moving into the next decade, the more densely sampled observing cadence of the Large Synoptic Survey Telescope (LSST; Ivezic et al. 2008) will provide even more data that can be used to characterize the behaviour of novae during the (potentially long-term) run-up to outburst. The arduous process of combing through archival plates in the hopes of serendipitously locating nova progenitors, and then processing those images to provide usable photometric data, will be increasingly supplanted by an ever-growing spatial and temporal database of accurate, homogeneous, digital photometry extending to very faint objects. This will finally allow us to systematically assess the presence and characteristics of photometric changes displayed by classical novae in relation to their outbursts, providing crucial input and constraints to modelling the physics behind these accretion-induced thermonuclear events.

Acknowledgements. The Pan-STARRS1 Surveys (PS1) have been made possible through contributions of the Institute for Astronomy, the University of Hawaii, the Pan-STARRS Project Office, the Max-Planck Society and its participating institutes, the Max Planck Institute for Astronomy, Heidelberg and the Max Planck Institute for Extraterrestrial Physics, Garching, The Johns Hopkins University, Durham University, the University of Edinburgh, Queen's University Belfast, the Harvard-Smithsonian Center for Astrophysics, the Las Cumbres Observatory Global Telescope Network Incorporated, the National Central University of Taiwan, the Space Telescope Science Institute, the National Aeronautics and Space Administration under Grant No. NNX08AR22G issued through the Planetary Science Division of the NASA Science Mission Directorate, the National Science Foundation under Grant No. AST-1238877, the University of Maryland, and Eotvos Lorand University (ELTE). This research has made use of data obtained from the SuperCOSMOS Sky Survey, prepared and hosted by the Wide Field Astronomy Unit, Institute for Astronomy, University of Edinburgh, which is funded by the UK Science and Technology Facilities Council. This research has made use of the APASS database, located at the AAVSO web site. Funding for APASS has been provided by the Robert Martin Ayers Sciences Fund. We thank our anonymous reviewer for a helpful and extremely swift referee report.

\section{References}

Blanton, M. R., \& Roweis, S. 2007, AJ, 133, 734

Bruch, A. 1992, A\&A, 266, 237

CMC 2006, Carlsberg Meridian Catalog 14 (CMC14) (Copenhagen University Obs., Institute of Astronomy, Cambridge, Real Instituto Y Observatorio de La Armada, Fernando En San)

Collazzi, A. C., Schaefer, B. E., Xiao, L., et al. 2009, AJ, 138, 1846

Darnley, M. J., \& Bode, M. F. 2013, ATel, 5300

Denisenko, D., \& Masi, G. 2013, IAU Circ., ed. D. W. E. Green, 9258

Drake, A. J., Djorgovski, S. G., Mahabal, A., et al. 2012, Proc. IAU, 285, 306

Hambly, N., Irwin, M., \& MacGillivray, H. 2001a, MNRAS, 326, 22

Hambly, N., MacGillivray, H., Read, M., et al. 2001b, MNRAS, 326, 1279

Hays, E., Cheung, T., \& Ciprini, S. 2013, ATel, 5302

Ivezic, Z., Axelrod, T., Brandt, W., et al. 2008, Serb. Astron. J., 176, 1

Jadhav, Y., Deacon, N., Magnier, E. A., Hoard, D. W., \& Huber, M. 2014, in AAS Meeting \#223, \#154.03

Kaiser, N., Aussel, H., Burke, B. E., et al. 2002, in Survey and Other Telescope Technologies and Discoveries, eds. S. Tyson, \& J. Anthony, \& S. Wolff, Proc. SPIE, 4836, 154

Keller, S. C., Schmidt, B. P., Bessell, M. S., et al. 2013, Publ. Astron. Soc. Aust., 24,1

Magnier, E. A., Schlafly, E., Finkbeiner, D., et al. 2013, ApJS, 205, 20

Morganson, E., De Rosa, G., Decarli, R., et al. 2012, AJ, 143, 142

Munari, U., \& Henden, A. 2013, Commissions 27 and 42 of the IAU information bulletin on variable stars, 6087

Page, K. L., Osborne, J. P., Kuin, N. P. M., et al. 2013, ATel, 5470

Rau, A., Kulkarni, S. R., Law, N. M., et al. 2009, PASP, 121, 1334

Robinson, E. L. 1975, AJ, 80, 515

Roy, N., Kantharia, N. G., Dutta, P., et al. 2013, ATel, 5376

Scaringi, S. 2014, MNRAS, 438, 1233

Schaefer, B. E. 2014, in AAS Meeting \#223, \#209.01

Schaefer, B. E., Landolt, A. U., Linnolt, M., et al. 2013, ApJ, 773, 55

Schlafly, E. F., Finkbeiner, D. P., Jurić, M., et al. 2012, ApJ, 756, 158

Stringfellow, G. S., Hebb, L., \& Fumagalli, M. 2013, ATel, 5336

Tang, S., Ofek, E. O., Kasliwal, M. M., et al. 2013, ATel, 5308

Tarasova, T. 2013, ATel, 5297

Tonry, J. L., Stubbs, C. W., Lykke, K. R., et al. 2012, ApJ, 750, 99

Waagen, E. O. 2013, AAVSO Alert Notice, 489

Warner, B. 2003, Cataclysmic Variable Stars (Cambridge: Cambridge University Press)

Wren, J., Vestrand, W. T., Wozniak, P., \& Davis, H. 2013, ATel, 5316 